\section{Mortalidade infantil segundo raça/cor no Brasil: o que dizem os sistemas nacionais de informação?}

\author{
Infant mortality according to race/color \\ in Brazil: what do the national databases say?
}

\author{
1 Centro de Vigilância \\ Epidemiológica, Secretaria \\ de Estado de Saúde \\ do Rio de Janeiro, \\ Rio de Janeiro, Brasil. \\ 2 Escola Nacional de Saúde \\ Pública, Fundação Oswaldo \\ Cruz, Rio de Janeiro, Brasil. \\ 3 Museu Nacional, \\ Universidade Federal \\ do Rio de Janeiro, \\ Rio de Janeiro, Brasil. \\ Correspondência \\ R. V. Santos \\ Escola Nacional de Saúde \\ Pública, Fundação Oswaldo \\ Cruz. Rua Leopoldo Bulhões \\ 1480, Rio de Janeiro, $R J$ \\ 21041-210, Brasil. \\ santos@ensp.fiocruz.br
}

\section{Abstract}

This study analyzes the consistency of the Brazilian national information systems on mortality (SIM) and live births (SINASC) as data sources for evaluating health inequalities according to race/color. Infant mortality rates (IMRs) were obtained according to race/color from death and live birth certificates for the country as a whole and its regions, for the years 1999-2002. The IMR was also estimated according to racel color, based on the incorporation of deaths and live births with race/color not reported by two criteria. The study compared the IMRs obtained in the study with those estimated by indirect methods. The IMR ratios were also calculated between race/color categories. A substantial reduction was observed during the period in the number of deaths and live births with race/color not recorded. In 2002, infant mortality in black children was $30.0 \%$ to $80.0 \%$ higher than that of white children and $40.0 \%$ to $80.0 \%$ higher than that of brown or mixed-race children (pardas); infant mortality in indigenous children was $40.0 \%$ to $90.0 \%$ higher than that of white or brown children. It is hoped that improved recording in the SIM and SINASC databases will allow a more in-depth discussion of health inequalities according to race, color, and ethnicity in Brazil.

Infant Mortality; Information Systems; Live Birth; Race; Skin Color
Andrey Moreira Cardoso 1 Ricardo Ventura Santos 2,3 Carlos E. A. Coimbra Jr. ${ }^{2}$

\section{Introdução}

Nos últimos anos vem se intensificando o interesse pelas análises acerca de raça/cor e etnia como fatores determinantes de desigualdades em saúde no Brasil 1,2,3,4,5,6. Conforme apontam alguns desses autores, dificuldade importante nessas análises relaciona-se à cobertura e à qualidade das bases de dados nacionais, cuja coleta de dados sobre raça/cor e etnia é relativamente recente e ainda deficiente.

Este trabalho tem por objetivo discutir a consistência do Sistema de Informações sobre Mortalidade (SIM) e do Sistema de Informações sobre Nascidos Vivos (SINASC) como fontes de dados para a avaliação sistemática das desigualdades raciais e étnicas em saúde, através da análise das taxas de mortalidade infantil (TMI).

\section{Metodologia}

Foram levantadas as freqüências de nascidos vivos e de óbitos infantis segundo raça/cor para o Brasil e grandes regiões, respectivamente por meio de consulta às bases de dados nacionais do SINASC e do SIM 7. A análise foi realizada para o período de 1999 a 2002. Esse período foi selecionado em decorrência de modificações na ordem das categorias do campo de raça/cor na Declaração de Óbito (DO) em 1998 (o que poderia resultar em alterações quanto à 
possibilidade da classificação de um indivíduo em uma dada categoria de raça/cor, comprometendo a comparabilidade dos dados anteriores e posteriores à modificação) e da ausência de dados consolidados pelo Ministério da Saúde após 2002.

As freqüências de óbitos e de nascidos vivos de cada categoria de raça/cor compuseram os numeradores e os denominadores das TMIs, segundo raça/cor informada nas DO e nas Declarações de Nascidos Vivos (DNV), para o país e grandes regiões. Dessa forma, a taxa de mortalidade infantil informada (TMIinfo) para uma determinada categoria de raça/cor foi calculada pela razão entre o número de óbitos em menores de um ano com raça/cor informada pertencente àquela categoria e o número de nascidos vivos (NV) com raça/cor informada na mesma categoria, multiplicada por mil.

Devido à elevada proporção de não informação sobre raça/cor nas DO e DNV, foram estimadas variantes de TMI segundo raça/cor a partir da incorporação dos óbitos e NV com raça/cor não informada, considerando duas possibilidades: (1) assumindo que a probabilidade de não informação de uma dada categoria de raça/cor não difere das demais, estimou-se a TMI a partir da distribuição dos óbitos e NV com raça/cor não informada proporcionalmente aos respectivos números de óbitos e NV informados por categoria de raça/cor (TMI proporcionalmente modificada - TMIprop); (2) assumindo que a não informação sobre raça/cor se restringe apenas a uma das categorias de raça/cor, estimou-se a TMI a partir da atribuição de todos os óbitos e nascimentos com raça/cor não informada à categoria de raça/cor sob análise (TMI modificada por raça/cor - TMImod). Para a TMIprop, a atribuição dos nascimentos e óbitos com raça/cor não informada às diversas categorias de raça/cor foi realizada por Unidade Federada (UF) em decorrência da heterogeneidade das proporções de não informação. Posteriormente, agregaram-se os números absolutos para cálculo das TMIprop do país e das grandes regiões.

Analisou-se o comportamento das TMI obtidas com base nos procedimentos descritos acima, comparando-as com as TMI estimadas por métodos indiretos, segundo Simões ( $A$ Mortalidade Infantil na Década de 90 e Alguns Condicionantes Sócio-econômicos. Rio de Janeiro: Instituto Brasileiro de Geografia e Estatística; 2003. mimeo.). Em seguida, foram calculadas as razões entre as TMI das crianças pardas, pretas e indígenas e as das crianças brancas, consideradas como categoria de referência, no período de 1999 a 2002. As crianças amarelas não foram incluídas nessa comparação devido ao fato de que, como veremos adiante, há possivelmente problema de registro para um dos anos considerados.

\section{Resultados}

A análise do SIM demonstra uma redução progressiva dos óbitos infantis no país, da ordem de 15,0\% entre 1999 (69.345) e 2002 (58.915), acompanhada por redução acentuada do número de óbitos com raça/cor não informada na DO. O total de óbitos infantis com raça/cor não informada passou de 26.710 (38,5\%) em 1999 para $15.216(25,8 \%)$ em 2002, correspondendo a uma redução de 43,0\% (Tabela 1). A proporção de óbitos infantis com raça/cor não informada é resultante de distintas qualidades de registro entre UF e grandes regiões. Apesar de todas as regiões demonstrarem redução das proporções de não registro dessa variável no período, o Nordeste e o Sudeste representam, respectivamente, $40,2 \%$ e $21,3 \%$ dos óbitos infantis com raça/cor não informada em 2002. O Nordeste, que possui a pior qualidade da informação sobre raça/cor na DO, apresenta proporção de sub-registro de raça/cor aproximadamente duas vezes maior do que o Sudeste e cinco vezes maior que o Sul, que teve a menor proporção de óbitos infantis sem registro de raça/cor no período (8,8\% em 2002). A maior redução de não informação sobre raça/cor na DO nos quatro anos de análise foi observada no Centro-oeste (de 52,0\% em 1999 para 17,4\% em 2002).

Quanto ao SINASC, houve uma redução da ordem de $6,0 \%$ no número absoluto de NV entre 1999 (3,25 milhões) e 2002 (3,05 milhões). A redução da proporção de NV com raça/cor não informada no período foi expressiva, tendo caído de 920.252 (28,3\%) em 1999 para 360.417 (11,8\%) em 2002, o que corresponde a uma redução de 58,3\%. Nota-se que, tal como no caso da mortalidade, há heterogeneidade entre as UF e as grandes regiões (Tabela 1).

As TMIinfo, TMIprop e TMImod por categoria de raça/cor e ano estão apresentadas na Tabela 2. De forma geral, no período 1999/2002 observa-se uma tendência levemente decrescente das TMI obtidas pelos três métodos para brancos e pardos, que apresentam TMI seme- 
Proporção (\%) de óbitos infantis e nascidos vivos com raça/cor não informada segundo macrorregiões e Unidade Federada (UF). Brasil, 1999/2002.

\begin{tabular}{|c|c|c|c|c|c|c|c|c|}
\hline \multirow[t]{2}{*}{ Brasil e macrorregiões } & \multicolumn{4}{|c|}{ Óbitos (\%) } & \multicolumn{4}{|c|}{ Nascidos vivos (\%) } \\
\hline & 1999 & 2000 & 2001 & 2002 & 1999 & 2000 & 2001 & 2002 \\
\hline Rio Grande do Sul & 16,8 & 10,2 & 7,4 & 5,7 & 13,5 & 0,5 & 0,3 & 0,2 \\
\hline Paraná & 22,8 & 13,2 & 11,0 & 8,3 & 23,9 & 2,4 & 3,2 & 1,9 \\
\hline Santa Catarina & 30,7 & 17,4 & 15,2 & 15,7 & 21,3 & 2,5 & 0,5 & 0,4 \\
\hline Sul & 22,3 & 13,0 & 10,5 & 8,8 & 19,2 & 1,7 & 1,5 & 0,9 \\
\hline Roraima & 11,3 & 14,4 & 8,6 & 5,2 & 0,0 & 0,3 & 0,3 & 0,1 \\
\hline Amapá & 9,2 & 29,6 & 7,9 & 8,2 & 0,4 & 0,7 & 0,2 & 0,2 \\
\hline Pará & 31,2 & 28,5 & 21,4 & 8,4 & 3,8 & 0,9 & 0,5 & 0,2 \\
\hline Tocantins & 30,0 & 14,4 & 16,7 & 10,4 & 5,0 & 1,4 & 2,8 & 0,5 \\
\hline Amazonas & 18,1 & 13,5 & 7,7 & 13,6 & 6,3 & 6,8 & 2,4 & 1,1 \\
\hline Acre & 68,7 & 34,5 & 30,1 & 29,0 & 15,0 & 5,9 & 4,8 & 2,8 \\
\hline Rondônia & 51,2 & 41,3 & 27,4 & 29,8 & 5,6 & 2,0 & 2,0 & 2,0 \\
\hline Norte & 30,0 & 24,9 & 17,8 & 12,9 & 4,9 & 2,7 & 1,5 & 0,8 \\
\hline Mato Grosso do Sul & 59,4 & 21,1 & 9,6 & 6,4 & 10,0 & 1,8 & 0,6 & 0,4 \\
\hline Mato Grosso & 52,1 & 17,5 & 20,7 & 10,1 & 3,8 & 0,6 & 0,5 & 0,5 \\
\hline Distrito Federal & 27,4 & 25,2 & 18,8 & 16,2 & 22,6 & 28,5 & 29,6 & 35,1 \\
\hline Goiás & 59,0 & 33,5 & 31,2 & 28,4 & 29,4 & 15,0 & 12,7 & 14,6 \\
\hline Centro-oeste & 52,0 & 25,2 & 21,5 & 17,4 & 19,1 & 12,4 & 11,5 & 13,3 \\
\hline São Paulo & 19,8 & 10,8 & 7,9 & 8,1 & 29,2 & 25,6 & 24,9 & 23,9 \\
\hline Rio de Janeiro & 24,7 & 19,4 & 22,7 & 26,3 & 24,6 & 6,5 & 4,3 & 5,0 \\
\hline Minas Gerais & 44,3 & 34,2 & 36,9 & 34,3 & 51,3 & 14,6 & 14,7 & 14,3 \\
\hline Espírito Santo & 54,5 & 59,4 & 70,2 & 64,7 & 7,4 & 2,4 & 1,7 & 3,7 \\
\hline Sudeste & 28,4 & 20,8 & 21,4 & 21,3 & 32,3 & 18,2 & 17,3 & 17,0 \\
\hline Maranhão & 44,3 & 37,0 & 24,6 & 19,1 & 72,1 & 21,3 & 16,0 & 13,7 \\
\hline Pernambuco & 41,4 & 30,0 & 24,5 & 20,4 & 7,2 & 2,2 & 2,8 & 2,5 \\
\hline Paraíba & 70,4 & 47,4 & 39,0 & 40,1 & 43,3 & 13,0 & 11,3 & 8,6 \\
\hline Bahia & 67,7 & 51,4 & 47,0 & 44,3 & 49,5 & 20,8 & 18,6 & 21,8 \\
\hline Ceará & 54,4 & 54,2 & 49,8 & 45,0 & 30,2 & 11,4 & 12,4 & 11,6 \\
\hline Alagoas & 54,6 & 46,6 & 50,7 & 45,6 & 11,3 & 2,1 & 1,5 & 1,0 \\
\hline Piauí & 44,9 & 45,3 & 55,8 & 56,6 & 41,4 & 14,7 & 16,3 & 17,3 \\
\hline Sergipe & 82,5 & 80,6 & 71,0 & 63,0 & 52,9 & 43,0 & 46,4 & 43,9 \\
\hline Rio Grande do Norte & 37,4 & 51,9 & 76,9 & 72,6 & 27,7 & 1,5 & 1,1 & 0,9 \\
\hline Nordeste & 55,2 & 46,8 & 43,9 & 40,2 & 36,5 & 13,7 & 12,8 & 13,0 \\
\hline Brasil & 38,5 & 30,0 & 28,0 & 25,8 & 28,3 & 12,8 & 11,9 & 11,8 \\
\hline
\end{tabular}

Fonte: Ministério da Saúde 7.

lhantes e inferiores àquelas de pretos e indígenas. As categorias preta e, principalmente a indígena, apresentaram TMIinfo e TMIprop com tendência crescente, mas TMImod crescente de 1999/2000 e decrescente a partir de 2000. As crianças classificadas como amarelas apresentam as mais baixas TMIinfo e TMIprop para todos os anos, com exceção do ano de 2000, que possivelmente apresenta problemas de regis- tro. Já a TMImod das crianças amarelas aproxima-as das pretas e indígenas.

A comparação das TMI obtidas segundo os procedimentos explicitados na metodologia e aquelas obtidas por estimativas indiretas com base nos dados do Censo Demográfico 2000 do Instituto Brasileiro de Geografia e Estatística (IBGE) é apresentada na Tabela 3. A TMIinfo e a TMIprop apresentam valores subestimados 
em relação àqueles do IBGE, enquanto a TMImod tende a ser discretamente superestimada, com exceção da raça/cor parda e para o Brasil como um todo, para os quais todos os valores são subestimados. As TMI obtidas baseando-se nos registros do SIM e do SINASC para a raça/cor branca são as mais próximas da TMI estimada pelo IBGE. Por sua vez, a raça/cor parda apresentou as TMI mais subestimadas.

A Figura 1 mostra a comparação das razões entre as TMI de pardos/brancos, pretos/brancos e indígenas/brancos no período 1999/2002. Observa-se que as crianças pardas apresentam TMI razoavelmente próximas das de crianças brancas. Já as pretas e indígenas apresentam TMI superiores às de crianças brancas, sendo que os diferenciais são crescentes no período quando se analisam as razões das TMIinfo e TMIprop. Em 2002, a mortalidade infantil das crianças pretas superou em $30,0 \%$ (TMIinfo) e $80,0 \%$ (TMImod) a mortalidade infantil das brancas. $\mathrm{O}$ diferencial de mortalidade entre crianças indígenas e brancas variou de 40,0\% (TMIprop) a 90,0\% (TMImod) a mais para as primeiras em 2002.

Tabela 2

Taxa de mortalidade infantil segundo raça/cor e ano de ocorrência. Brasil, 1999/2002.

\begin{tabular}{|c|c|c|c|c|c|c|c|c|c|c|c|c|}
\hline \multirow[t]{2}{*}{ Raça/cor } & \multicolumn{4}{|c|}{ TMlinfo* } & \multicolumn{4}{|c|}{ TMIprop** } & \multicolumn{4}{|c|}{ 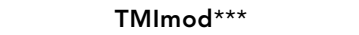 } \\
\hline & 1999 & 2000 & 2001 & 2002 & 1999 & 2000 & 2001 & 2002 & 1999 & 2000 & 2001 & 2002 \\
\hline Amarela & 14,9 & 28,5 & 10,3 & 11,1 & 17,0 & 33,2 & 12,1 & 10,6 & 27,6 & 49,0 & 45,0 & 41,0 \\
\hline Branca & 18,6 & 17,6 & 17,2 & 16,6 & 20,5 & 20,5 & 19,8 & 18,7 & 22,9 & 24,3 & 23,1 & 21,7 \\
\hline Parda & 17,6 & 15,8 & 14,7 & 15,3 & 22,5 & 21,7 & 19,5 & 19,4 & 23,5 & 25,0 & 22,6 & 21,7 \\
\hline Preta & 24,5 & 20,3 & 21,3 & 22,3 & 28,5 & 26,6 & 27,6 & 28,0 & 28,7 & 44,7 & 42,2 & 38,8 \\
\hline Indígena & 19,3 & 18,9 & 19,9 & 25,0 & 21,7 & 22,4 & 22,4 & 26,9 & 28,9 & 48,7 & 45,6 & 41,5 \\
\hline Global & 18,2 & 17,1 & 16,2 & 16,2 & 21,3 & 21,3 & 19,9 & 19,2 & 21,3 & 21,3 & 19,9 & 19,3 \\
\hline
\end{tabular}

Fonte: Ministério da Saúde 7

TMlinfo = taxa de mortalidade infantil informada; TMlprop = taxa de mortalidade infantil proporcionalmente modificada;

TMImod = taxa de mortalidade infantil modificada por raça/cor.

* As taxas de mortalidade infantil foram calculadas exclusivamente com os óbitos (numerador) e nascidos vivos (denominador)

com raça/cor informada na DO e na DNV, respectivamente;

** As taxas de mortalidade infantil proporcionalmente modificadas foram calculadas somando-se aos óbitos (numerador) e aos nascidos vivos (denominador) com raça/cor informada, respectivamente os óbitos e nascidos vivos com raça/cor

não informada em quantidade correspondente à distribuição proporcional daqueles informados, segundo UF de ocorrência;

$\star \star \star$ As taxas de mortalidade infantil modificadas por raça/cor foram calculadas somando-se aos óbitos (numerador) e aos

nascidos vivos (denominador), de cada raça/cor informada, o número total de óbitos e nascidos vivos com raça/cor não informada.

Comparação das taxas de mortalidade infantil por distintos métodos e fontes de dados segundo raça/cor. Brasil, 2000.

\begin{tabular}{lcccc}
\hline Raça/cor & $\begin{array}{c}\text { Informada - MS } \\
\text { (Razão MS/IBGE) }\end{array}$ & $\begin{array}{c}\text { Proporcionalmente } \\
\text { modificada - MS } \\
\text { (Razão MS/IBGE) }\end{array}$ & $\begin{array}{c}\text { Modificada por } \\
\text { raça/cor - MS } \\
\text { (Razão MS/IBGE) }\end{array}$ & IBGE 2000* \\
\hline Amarela & $28,5(-)$ & $33,2(-)$ & $49,0(-)$ & - \\
Branca & $17,6(0,77)$ & $20,5(0,89)$ & $24,3(1,06)$ & 22,9 \\
Parda & $15,8(0,48)$ & $21,7(0,66)$ & $25,0(0,76)$ & 33,0 \\
Preta & $20,3(0,58)$ & $26,6(0,76)$ & $44,7(1,28)$ & 34,9 \\
Indígena & $18,9(-)$ & $22,4(-)$ & $48,7(-)$ & - \\
Brasil & $17,1(0,58)$ & $21,3(0,72)$ & $21,3(0,72)$ & 29,7 \\
\hline
\end{tabular}

MS = Ministério da Saúde; IBGE = Instituto Brasileiro de Geografia e Estatística

* Censo Demográfico 2000 (IBGE), estimativas indiretas (Simões CCS. A Mortalidade Infantil

na Década de 90 e Alguns Condicionantes Sócio-econômicos. Rio de Janeiro: IBGE; 2003. mimeo.). 


\section{Figura 1}

Razão entre taxas de mortalidade infantil (TMI) informadas, TMI proporcionalmente modificadas e TMI modificadas. Brasil, 1999/2002.

Figura $1 \mathrm{a}$

Pardos e brancos

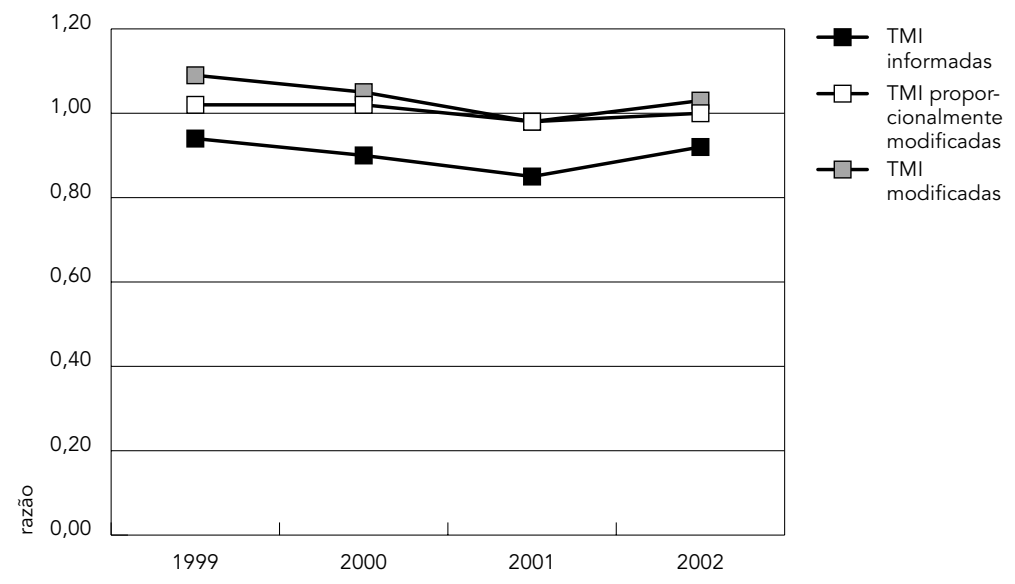

Figura $1 b$

Pretos e brancos

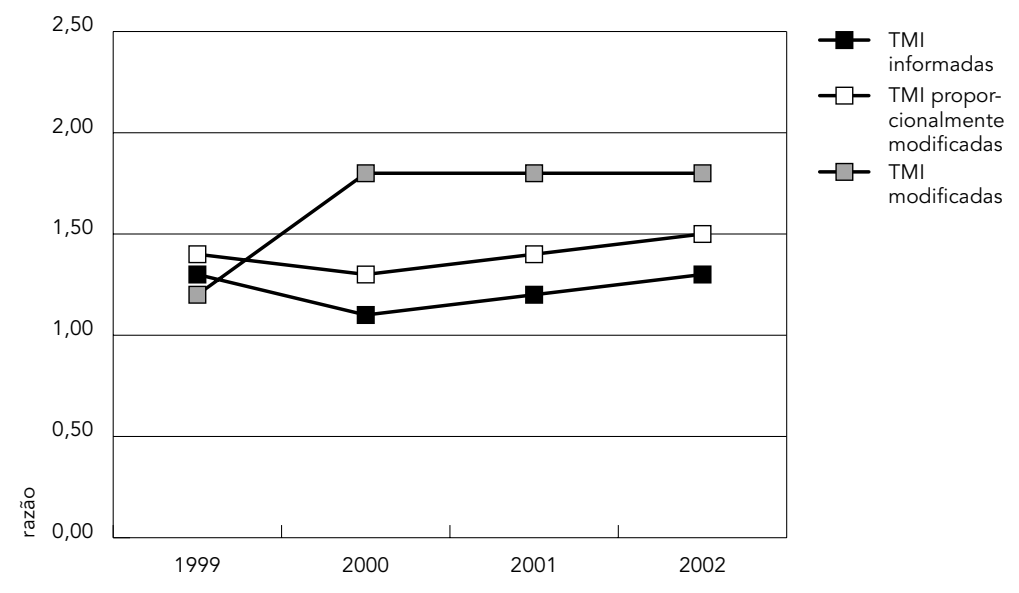

(continua)

\section{Discussão}

Conforme amplamente reconhecido, a categoria raça/cor é uma variável de definição e mensuração particularmente complexa 3,5,8,9. No caso específico da conformação de bases de estatísticas vitais (nascimentos e óbitos), são bastante óbvios os limites para a classificação da raça/cor de recém-nascidos e de cadáveres, inclusive pela impossibilidade de aplicação do procedimento mais amplamente recomendado, qual seja, a autoclassificação.

A disponibilidade e a qualidade das estatísticas vitais permanecem como fatores que limitam os estudos sobre a mortalidade no Brasil, principalmente quando analisada sob aspectos específicos, como raça/cor. Ainda que reconhecendo a ocorrência de sub-registro de óbitos, Vasconcelos 10 demonstra que tem havido aumento na cobertura do SIM, mesmo que de forma heterogênea entre regiões, áreas rurais e urbanas e segundo algumas características do falecido, como a idade. Nas regiões Norte, Nordeste e Centro-oeste observam-se menores níveis de cobertura do SIM.

Segundo análises recentes, nos últimos anos tem se verificado melhoria progressiva e significativa nas coberturas do SIM e do SINASC 10,11. Não obstante, o sub-registro de óbitos ainda permanece no patamar de $20,0 \%$ para a média nacional e em torno de $40,0 \%$ para as regiões Norte e Nordeste, sendo mais significativo em menores de um ano e em idosos. A cobertura do SINASC foi estimada em 93,0\% do total de nascidos vivos no país em 2002, com cobertura média de $75,0 \%$ no Norte e Nordeste 11.

De forma geral, além de aumento na cobertura, houve também uma melhoria no registro das variáveis contidas nas declarações de NV e óbitos no país. Ao longo do tempo, diminuiu o número de declarações sem registro de raça/cor, sobretudo nas DNV. Contudo, em 2002, $25,8 \%$ dos óbitos infantis ainda não tinham registro de raça/cor e, no caso dos NV, 11,8\% não apresentavam informação sobre essa variável. A melhoria da informação foi heterogênea entre as UF e grandes regiões. O país como um todo e as diversas regiões vêm apresentando progressiva redução nas proporções de NV e óbitos com raça/cor não informada. No Nordeste e no Centro-oeste, nota-se, contudo, ligeiro aumento na proporção de NV com raça/cor não informada em 2002. Nessas duas regiões pode estar havendo uma maior captação de nascimentos e óbitos, não acompanhada pela melhoria do registro de raça/cor em igual magnitude. Para a Região Norte que, ao contrário do esperado, se assemelha ao padrão do Sul e Su- 
deste, pode estar havendo manutenção do subregistro de nascimentos e óbitos, possivelmente nas áreas rurais, e melhoria da qualidade dos registros de raça/cor nas localidades onde nascimentos e óbitos são registrados, possivelmente nas áreas urbanas. É surpreendente a elevada proporção de sub-registro da variável raça/cor no Sudeste, onde pode-se observar UF representativas dos melhores (São Paulo) e dos piores registros de raça/cor do país (Espírito Santo), como evidência de desigualdades intra-regionais nos registros de óbitos e NV. O Sul invariavelmente chama a atenção para um registro mais completo das estatísticas vitais.

As diferenças de cobertura entre o SIM e o SINASC, mesmo com as melhorias observadas nos últimos anos, têm implicações importantes na análise da mortalidade infantil. Devido aos diferenciais de cobertura, as TMI obtidas valendo-se dessas bases são subestimadas em relação à realidade. A subestimação fica evidente ao se comparar as TMI por raça/cor geradas a partir dessas bases de dados com as TMI estimadas pelo IBGE para as mesmas categorias. Considerando esses fatos, os dados do SIM e do SINASC apresentam limitações para a caracterização dos diferenciais de mortalidade infantil segundo categorias de raça/cor no país.

Além da TMI diretamente obtida com base nos dados disponíveis no SIM e no SINASC (TMIinfo), neste trabalho estimaram-se duas outras taxas (TMIprop e TMImod) devido à elevada proporção de não informação sobre raça/cor nas DO e DNV. Baseando-se nos resultados tornou-se evidente que a TMImod apresenta problemas, pois, ao se alocar todos os óbitos e nascimentos para cada uma das categorias de raça/cor, há uma superestimação das TMI no caso das categorias com menor freqüência. Esse padrão é particularmente evidente para as crianças amarelas que, ainda que apresentando as menores TMIinfo e TMIprop, aproximam-se das pretas e indígenas ao se considerar a TMImod.

Mesmo diante das restrições mencionadas acima quanto aos sistemas de informação, ligadas à qualidade e à quantidade dos registros de NV e óbitos, foram detectadas desigualdades nas TMI segundo raça/cor que parecem espelhar o cenário maior de desigualdades sócioeconômicas e ambientais que caracterizam as relações raciais e étnicas no país 1,2,6,12. Ainda que os dados do SIM e do SINASC resultem em TMI subestimadas, crianças pretas e indígenas apresentam níveis de mortalidade infantil substancialmente superiores aos observados nas demais categorias de raça/cor.
Figura 1c (continuação)

Indígenas e brancos

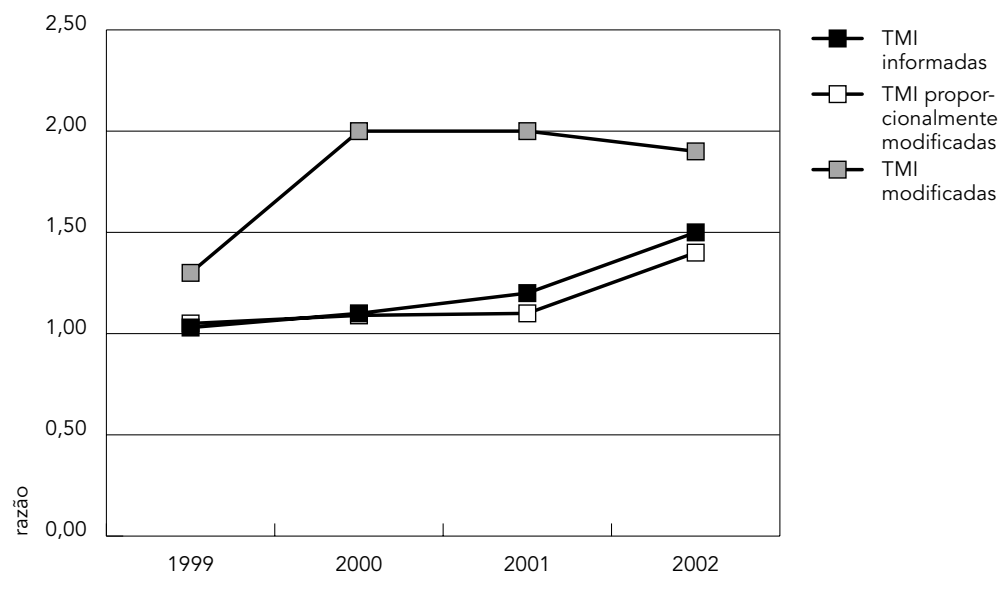

\section{Considerações finais}

O preenchimento da variável raça/cor nas DNV e DO apresenta problemas. Isso se relaciona não somente às dificuldades inerentes à classificação dos indivíduos, como também da inclusão relativamente recente dessa variável nos sistemas de informação. Essas dificuldades, além do sub-registro de óbitos e NV, impõem limites à análise e interpretação das TMI por raça/cor no país.

Conforme evidenciamos neste trabalho, a obtenção da TMI por método direto com base nos bancos do SIM e do SINASC tem valor limitado para estimar o risco de morte infantil segundo raça/cor, devido à heterogeneidade da cobertura e à qualidade dos registros entre unidades da federação e grandes regiões. Ainda assim, foram detectadas desigualdades raciais/étnicas na mortalidade infantil, chamando atenção que as crianças indígenas e pretas apresentam comparativamente as mais elevadas TMI. A melhoria dos registros do SIM e do SINASC que vem ocorrendo, e que se espera que persista, trará subsídios mais concretos para se discutir, e possivelmente confirmar, esses padrões de desigualdade, o que deve vir associado a análises que levem em consideração a influência de aspectos sócio-econômicos.

Consideramos prioritário que esforços sejam feitos no sentido de melhorar a cobertura e a qualidade das estatísticas vitais segundo raça/cor no país, com vistas a permitir o aprofundamento da discussão sobre desigualdades em saúde no país segundo raça, cor e etnia. 


\section{Resumo}

O trabalho analisa a consistência dos Sistemas de Informações Sobre Mortalidade (SIM) e Sobre Nascidos Vivos (SINASC) como fontes de dados para a avaliação de desigualdades em raça/cor em saúde no Brasil. Foram obtidas taxas de mortalidade infantil (TMI) segundo raça/cor informada nas declarações de óbitos e nascidos vivos (NV), para o país e grandes regiões, no período 1999/2002. Estimou-se também a TMI segundo raça/cor a partir da incorporação dos óbitos e NV com raça/cor não informada por dois critérios. Compararam-se as TMI obtidas no estudo com as TMI estimadas por métodos indiretos. Foram também calculadas razões das TMI entre categorias de raça/cor. Observou-se redução substancial do número de óbitos e NV com raça/cor não informada no período. Em 2002, a mortalidade infantil das crianças pretas superou em $30,0 \%$ e 80,0\% a mortalidade infantil das brancas e em 40,0\% e 80,0\% a das pardas; o diferencial de mortalidade entre crianças indígenas e brancas ou pardas variou de 40,0\% a 90,0\% a mais para as primeiras. Espera-se que a melhoria dos registros do SIM e do SINASC permita um aprofundamento da discussão sobre desigualdades em saúde segundo raça, cor e etnia no país.

Mortalidade Infantil; Sistemas de Informação; Nascidos Vivos; Raça; Cor da Pele

\section{Colaboradores}

A. M. Cardoso foi responsável pela análise e interpretação dos dados e pela redação do artigo. R. V. Santos e C. E. A. Coimbra Jr. participaram da interpretação dos dados e da redação do artigo.

\section{Agradecimentos}

A Nilza M. Pereira (Instituto Brasileiro de Geografia e Estatística) e Paulo Sabroza (Escola Nacional de Saúde Pública, Fundação Oswaldo Cruz) pelo interesse e disponibilidade para discutir os argumentos desenvolvidos no trabalho. Também a Eduardo Faerstein (Universidade do Estado do Rio de Janeiro) pela leitura crítica e comentários.

\section{Referências}

1. Coimbra Jr. CEA, Santos RV. Saúde, minorias e desigualdade: algumas teias de inter-relações, com ênfase nos povos indígenas do Brasil. Ciênc Saúde Coletiva 2000; 5:125-32.

2. Cunha EMGP. Infant mortality and race: the differences of inequality. In: Hogan DJ, editor. Population change in Brazil: contemporary perspectives. Campinas: MPC Artes Gráficas; 2001. p. 333-6.

3. Monteiro SS. Desigualdades em saúde, raça e etnicidade: questões e desafios. In: Monteiro SS, Sansone L, organizadores. Etnicidade na América Latina: um debate sobre saúde, raça e direitos reprodutivos. Rio de Janeiro: Editora Fiocruz; 2004. p. 45-56.

4. Olinto MTA, Olinto BA. Raça e desigualdade entre as mulheres: um exemplo no sul do Brasil. Cad Saúde Pública 2000; 16:1137-42.

5. Travassos C, Williams DR. The concept and measurement of race and their relationship to public health: a review focused on Brazil and the United States. Cad Saúde Pública 2004; 20:660-78.

6. Santos RV, Coimbra Jr. CEA. Cenários e tendências da saúde e da epidemiologia dos povos indígenas no Brasil. In: Coimbra Jr. CEA, Santos RV, Escobar AL, organizadores. Epidemiologia e saúde dos povos indígenas no Brasil. Rio de Janeiro: Editora Fiocruz; 2003. p. 13-47.

7. Ministério da Saúde. Banco de dados dos Sistemas de Informação sobre Mortalidade (SIM) e Nascidos Vivos (SINASC), 1996 a 2002. Série G. Estatística e informação em saúde [CD-ROM]. Brasília: Secretaria de Vigilância em Saúde, Departamento de Análise de Situação em Saúde; 2004.

8. Maio MC, Monteiro S, Chor D, Faerstein E, Lopes CS. Cor/raça no Estudo Pró-saúde: resultados comparativos de dois métodos de autoclassificação no Rio de Janeiro. Cad Saúde Pública 2005; 21:171-80.

9. Bastos FI, Travassos C. "Raça" e saúde pública: os dilemas da ciência e da prática contemporânea. In: Minayo MCS, Coimbra Jr. CEA, organizadores. Críticas e atuantes: ciências sociais e humanas em saúde na América Latina. Rio de Janeiro: Editora Fiocruz; 2005. p. 461-71.

10. Vasconcelos AMN. A qualidade das estatísticas de óbito no Brasil. Revista Brasileira de Estudos Populacionais 1998; 15:115-24.

11. Rede Interagencial de Informações para a Saúde. Indicadores básicos de saúde no Brasil: conceitos e aplicações. Brasília: Organização Pan-Americana da Saúde; 2002.

12. Telles E. Racismo à brasileira: uma nova perspectiva sociológica. Rio de Janeiro: Relume-Dumará; 2003.

Recebido em $27 / \mathrm{Jul} / 2005$

Versão final reapresentada em 15/Ago/2005

Aprovado em 15/Ago/2005 